Tropical Journal of Pharmaceutical Research October 2019; 18 (10): 2011-2017

ISSN: $1596-5996$ (print); 1596-9827 (electronic)

(C) Pharmacotherapy Group, Faculty of Pharmacy, University of Benin, Benin City, 300001 Nigeria.

\title{
MiR-498 suppresses proliferation and inflammation in fibroblast-like synoviocytes in rheumatoid arthritis via targeting JAK1
}

\author{
Lan Chai ${ }^{1}$, Xian Zhen Zhang ${ }^{2 \star}$, Hai fang $\mathrm{Ma}^{3}$, Fang Yuan ${ }^{1}$ \\ ${ }^{1}$ Department of Rheumatology and Immunology, Zhejiang Hospital, ${ }^{2}$ Department of Rheumatology, Tongde Hospital of Zhejiang \\ Province, ${ }^{3}$ Department of Clinical Laboratory, Zhejiang Hospital, Hang Zhou City, Zhe Jiang Province 310000, China
}

*For correspondence: Email: XianZhengZhang125@163.com; Tel: 0086+0571-89972412

\begin{abstract}
Purpose: To investigate the effect of microRNA 498 (miR-498) on proliferation and inflammation of rheumatoid arthritis (RA) fibroblast-like synoviocytes (RA-FLSs) in rheumatoid arthritis (RA).

Methods: MiR-498 level was evaluated in both RA synovial tissues and RA-FLSs using real-time polymerase chain reaction (PCR). MicroRNA-498 overexpression or knockdown was performed in RAFLSs. Proliferation, apoptosis, cell cycle and inflammation induced by miR-498 mimics or inhibitor were used to explore the function of miR-498 in RA.

Results: Expression level of miR-498 was downregulated in both RA synovial tissues and RA- FLSs. MicroRNA-498 mimics decreased proliferation and arrested cell cycle, whereas miR-498 inhibitor caused the opposite effects in RA-FLSs. In addition, miR-498 mimics suppressed inflammation and promoted cell apoptosis, while miR-498 inhibitor promoted inflammation and inhibited cell apoptosis in $R A-F L S s$. Furthermore, the effect of miR-498 on the proliferation, inflammation and apoptosis of RAFLSs was mediated by its ability to target and downregulate JAK1.

Conclusion: These results indicate that miR-498 inhibits the proliferation and inflammatory responses of RA-FLSs by targeting JAK1, thus revealing a new therapeutic target for RA treatment.
\end{abstract}

Keywords: Rheumatoid arthritis, MiR-498, JAK1, Proliferation, Inflammation

\begin{abstract}
This is an Open Access article that uses a fund-ing model which does not charge readers or their institutions for access and distributed under the terms of the Creative Commons Attribution License (http://creativecommons.org/licenses/by/4.0) and the Budapest Open Access Initiative (http://www.budapestopenaccessinitiative.org/read), which permit unrestricted use, distribution, and reproduction in any medium, provided the original work is properly credited.

Tropical Journal of Pharmaceutical Research is indexed by Science Citation Index (SciSearch), Scopus, International Pharmaceutical Abstract, Chemical Abstracts, Embase, Index Copernicus, EBSCO, African Index Medicus, JournalSeek, Journal Citation Reports/Science Edition, Directory of Open Access Journals (DOAJ), African Journal Online, Bioline International, Open-J-Gate and Pharmacy Abstracts
\end{abstract}

\section{INTRODUCTION}

Rheumatoid arthritis (RA), a chronic autoimmune disease, is characterized by inflammation and destruction of the joints $[1,2]$. It reduces the quality of life, produces loss of functionality, and increases morbidity and mortality [2]. The incidence of RA has reached $0.5-1 \%$ worldwide [3]. The main clinical manifestations involved in the symmetrical joint are arthralgia, redness, swelling, and limited range of motion [4]. Current RA treatment is focused on blocking inflammation early in the disease course [2]. In addition, the main goals of RA treatment are to reduce joint and organ damage, improve physical function, and prevent long-term complications [2]. Fibroblast-like synoviocytes (FLSs) in the synovial intimal lining play a crucial role in RA pathogenesis [5]. Therefore, exploring the mechanism underlying RA treatment in RA-FLSs 
is of great importance. MicroRNAs (miRNAs), small non-coding RNAs, are single-stranded RNAs of 19-25 nucleotides in length which cause mRNA degradation and protein activity reduction by binding to the 3 '-untranslated region (UTR) of the target mRNA [6-9]. MicroRNAs have been reported to be associated with RAFLSs. For example, miR-708-5p is downregulated in the synovial tissues of patients with RA. On the other hand, miR-708-5p induces cell apoptosis and suppresses colony formation and migration in RA-FLSs [10]. Moreover, the expression of miR-199a-3p is low in RA-FLSs, which results in decreased proliferation and elevated apoptosis [11]. Interestingly, miR-498 is associated with RA susceptibility [12], suggesting that miR-498 may be involved in RA-FLS progression. Janus kinase 1 (JAK1) is associated with the RA progression due to its role in cytokine signaling [13,14], and it regulates RAFLSs. Previous studies have found that apoptosis of RA-FLSs can be induced via the JAK1-mediated signaling cascade [15]. In addition, JAK1 is involved in the activation of RAFLSs in response to the inflammatory cytokines interleukin 6 and oncostatin M [16]. Although JAK1 is reported to have a role in RA, the functions of miR-498 and JAK1 in RA-FLSs have not been investigated. This study first demonstrated the effect of miR-498 on the proliferation and inflammation of RA-FLSs via targeting JAK1. Furthermore, this work will be useful to explore new therapeutic targets of RA.

\section{EXPERIMENTAL}

\section{Human samples}

Thirty-two RA synovial and normal tissues from Zhejiang Hospital were collected, and they were divided into two groups: a normal group $(n=10)$ and an RA group $(n=22)$. All patients provided informed consent. The research protocols were approved by the Clinical Research Ethics Committee of Zhejiang Hospital (Approval no.2017-c-076) and World Medical Association Declaration of Helsinki.

\section{Cell culture and transfection}

The RA-FLSs were isolated from RA synovial tissues and were grown in RPMI 1640 medium containing $10 \%$ fetal bovine serum (FBS), and 293T cells were grown in Dulbecco's modified eagle medium supplemented with $10 \%$ FBS. On the other hand, miR-498 mimics (miR-498), scrambled mimic control (Control), miR-498 inhibitor (miR-498-inh), and inhibitor negative control (NC-inh) were purchased from RiboBio (Guangzhou, China). The RA-FLSs were then transfected with these RNAs using Lipofectamine 2000 (Life Technologies Corp., Carlsbad, CA, USA). All cells were cultured in a humidified incubator with $5 \% \mathrm{CO}_{2}$ at $37^{\circ} \mathrm{C}$.

\section{Quantitative real-time polymerase chain reaction (qPCR)}

Total RNA from RA synovial tissues or RA-FLSs was extracted using TRIzol (Invitrogen, Carlsbad, CA, USA). The RNA was then converted to cDNA using the universal cDNA synthesis kit II (Exiqon, Vedbaek, Denmark). SYBR Green (Takara Bio, Shiga, Japan) was used to perform qPCR. The primers used were as follows: miR498: forward:

5'-

TTTCAAGCCAGGGGGCGTTTTTTC-3', reverse: 5'-GCTTCAAGCTCTGGAGGTGCTTTTTC-3'.

Amplification of U6 was used as the control.

\section{Cell proliferation assay}

Cell proliferation was assessed using MTT assay kit. Cells of $2 \times 10^{4}$ RA-FLSs were first seeded into 96-well plates. Thereafter, $10 \mu \mathrm{L}$ of MTT solution was added (Dojindo Molecular Technologies, Gaithersburg, MD) per well at different periods of time, followed by incubation for another hour at $37^{\circ} \mathrm{C}$. The absorbance was measured at each time point using a microplate reader.

\section{Western blotting}

Proteins from RA-FLSs were extracted and then quantified using BCA Protein Assay Kit (Pierce, Appleton, WI, USA). Equal amounts of proteins were separated using sodium dodecyl sulfatepolyacrylamide gel electrophoresis and then transferred onto polyvinylidene fluoride membranes (Millipore, Bedford, MA, USA). The membranes were then blocked using blocking buffer and incubated with specific primary antibodies overnight at $4{ }^{\circ} \mathrm{C}$. Next, the membranes were incubated with horseradish peroxidase (HRP)-conjugated secondary antibodies for $1 \mathrm{~h}$ at $37^{\circ} \mathrm{C}$, followed by visualization of the bands. The corresponding primary antibodies used were: Cyclin D1 (1:1000; Santa Cruz Biotechnology, CA, USA), p27 (1:1000; Santa Cruz Biotechnology, CA, USA), Bcl-2 (1:1000; Santa Cruz Biotechnology, CA, USA), Bax (1:500; Abcam, Cambridge, MA, USA), cleaved caspase-3 (1:500; Abcam, Cambridge, MA, USA), JAK1 (1:1000; Santa Cruz Biotechnology, CA, USA), and $\beta$-actin $(1: 1000$; Cell Signaling Technology, Houston, TX, USA). 


\section{Cell cycle analysis}

The transfected cells were collected and washed thrice with cold phosphate-buffered saline (PBS). Next, they were re-suspended in pre-cooled $75 \%$ ethanol and incubated overnight at $4{ }^{\circ} \mathrm{C}$ for fixation. The ethanol was then discarded, and $500 \mu \mathrm{l}$ of PBS containing $20 \mu \mathrm{l}$ of RNase A (100 $\mu \mathrm{g} / \mathrm{mL}$ ) was added. After incubation for $30 \mathrm{~min}$, $400 \mu \mathrm{l}$ of propidium iodide (PI; $50 \mu \mathrm{g} / \mathrm{mL}$ ) was added to the cells drop-wise, followed by incubation for another $30 \mathrm{~min}$. The cell cycle distribution was evaluated using FACScan flow cytometry (BD Biosciences, San Jose, CA, USA).

\section{Cell apoptosis assay}

Cell apoptosis was measured using the Annexin V-FITC/PI apoptosis detection kit (BD Pharmingen, San Jose, CA, USA). The cells were collected after transfection with miR-498 mimics or miR-498 inhibitor. They were then washed and re-suspended with $100 \mu \mathrm{L}$ of binding buffer. Next, Annexin V-fluorescein isothiocyanate (FITC) and PI were added. After incubation for $15 \mathrm{~min}$, the stained cells were detected using FACScan flow cytometry (BD Biosciences, San Jose, CA, USA).

\section{Luciferase reporter assay}

The JAK1 and miR-498 binding sites were predicted using TargetScan software. The fragment of wild-type or mutant JAK1 was integrated into the pmir-GLO reporter vector (pmirGLO-JAK1-wt and pmirGLO-JAK1-mut). The luciferase activities were detected after cotransfection with miRNAs (miR-498 mimics, scrambled mimic control, miR-498 inhibitor, or inhibitor negative control) and reporter vectors (pmirGLO-JAK1-wt and pmirGLO-JAK1-mut) for $48 \mathrm{~h}$.

\section{Statistical analysis}

Data are reported as mean \pm standard deviation (SD). Student's t-test was used to analyze differences between two groups. One-way analysis of variance (ANOVA) followed by Tukey's test was applied for multiple comparisons, and $p<0.05$ was considered statistically significant.

\section{RESULTS}

\section{Expression of miR-498 in RA synovial tissues and RA-FLSs}

To examine miR-498 expression, the RA samples were harvested. As shown in Table 1, patients with RA were characterized based on parameters such as age, sex, duration of symptoms, rheumatoid factor (RF) positivity, anticyclic citrullinated peptide (anti-CCP) titer, and disease activity score 28 (DAS28). Next, miR498 expression was evaluated using real-time PCR. The results showed that miR-498 was significantly down-regulated in RA synovial tissues when compared with that in normal tissues (Figure $1 \mathrm{~A}, p<0.05)$. Similarly, miR-498 was decreased in RA-FLSs compared with that in FLSs (Figure $1 \mathrm{~B}, p<0.05$ ). Thus, the expression of miR-498 is inhibited in RA synovial tissues and RA-FLSs.

Table 1: Characteristics of RA patients

\begin{tabular}{lc}
\hline Parameter & Value \\
\hline Age, mean \pm SD (years) & $46 \pm 15.5$ \\
Sex, male/female & $6 / 16$ \\
Duration (months) of symptoms, & $57.66 \pm 50.20$ \\
mean \pm SD & \\
RF positive & $80.89 \%$ \\
RF titer, median units (range) & $116.2(20-1050)$ \\
ESR, mean \pm SD (mm/h) & $65.66 \pm 20.17$ \\
CCP titer, median units (range) & $72.3(13700)$ \\
Stiffness, median min (range) & $75.43 \pm 89.21$ \\
Treatment & \\
Etanercept, \% ( $n)$ & $68 \%(15)$ \\
Adalimumab, \% $(n)$ & $32 \%(s 7)$ \\
DAS28(ESP) score, mean \pm SD & $6.13 \pm 1.35$ \\
\hline
\end{tabular}

A

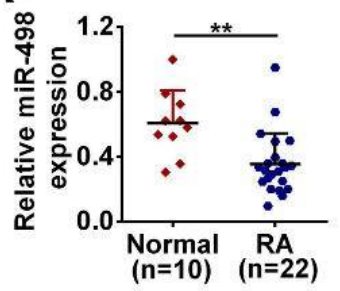

B

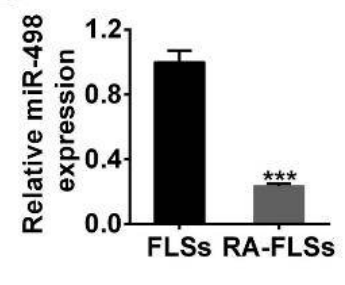

Figure 1: Expression of miR-498 in RA synovial tissues and FLSs. (A) Real-time PCR detected the decrease in miR-498 expression in RA synovial tissues compared with that in normal tissues. (B) Realtime PCR revealed that miR-498 expression was significantly decreased in RA-FLSs compared with that in normal FLSs; ${ }^{* *} p<0.01,{ }^{* * *} p<0.001$

\section{MiR-498 inhibits proliferation in RA-FLSs}

To further investigate the function of miR-498 in RA-FLS proliferation, this study carried out miR498 overexpression and knockdown experiments. First, real-time PCR indicated that miR-498 mimics and inhibitor were successfully expressed in RA-FLSs. The relative levels of miR-498 due to transfection with mimics were significantly increased compared with that of the Control (Figure 2A, $p<0.05$ ). Conversely, the introduction of miR-498 inhibitor in the RA-FLSs significantly reduced the expression of miR-498 
when compared with the introduction of NC-inh (Figure $2 \mathrm{~A}, p<0.05$ ). Next, the MTT assay revealed that miR-498 mimics decreased proliferation, whereas miR-498 inhibitor increased proliferation in RA-FLSs (Figure $2 \mathrm{~B}$ ). Western blot assay further demonstrated that miR-498 mimics decreased the level of Cyclin D1 and increased the level of p27 in RA-FLSs. However, miR-498 inhibitor induced the reverse effect on the Cyclin D1 and p27 protein levels (Figure $2 \mathrm{C}$ ). Furthermore, to study the cell cycle distribution of RA-FLSs, cell cycle profiles of miR-498 mimics or inhibitor cells were measured using flow cytometry. As shown in Figure $2 \mathrm{D}$, miR-498 mimics increased the percentage of cells in the G1 phase and decreased the percentage of cells in the $S$ phase, while miR498 inhibitor decreased the percentage of $\mathrm{G} 1$ cells and augmented the percentage of $S$ cells. Thus, miR-498 inhibits the proliferation of RAFLSs.

A
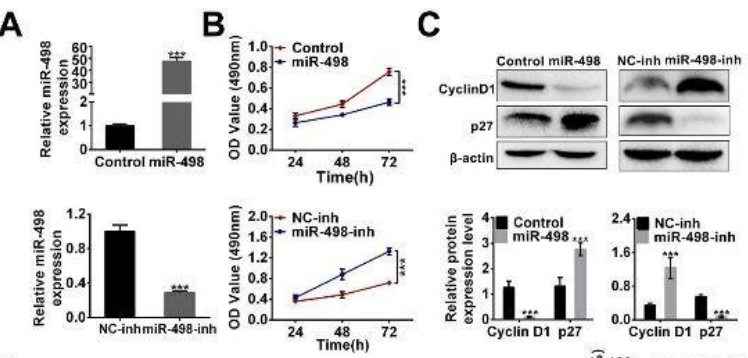

D

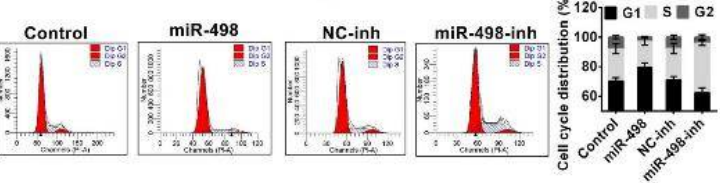

Figure 2: Effects of miR-498 on the proliferation of RA-FLSs. (A) Real-time PCR was used to evaluate the efficiency of miR-498 mimics or inhibitor in RA-FLSs. (B) MiR-498 mimics decreased proliferation, whereas miR-498 inhibitor increased proliferation in RA-FLSs using the MTT assay. (C) Western blotting revealed that miR-498 mimics inhibited Cyclin D1 protein level and enhanced the p27 protein level. However, miR498 inhibitor increased Cyclin D1 protein level and suppressed the p27 protein level in RA-FLSs. (D) MiR498 mimics increased the percentage of cells in the G1 phase and decreased the percentage of cells in the S phase, whereas miR-498 inhibitor decreased the percentage of $\mathrm{G} 1$ cells and augmented the percentage of $S$ cells; ${ }^{* * *} \underline{p}<0.001$

\section{MiR-498 suppress inflammation and promote apoptosis in RA-FLSs}

This work next detected whether miR-498 could affect inflammation and apoptosis in RA-FLSs. Enzyme-linked immunosorbent assay (ELISA) showed that miR-498 mimics suppressed TNF- $\alpha$ and IL-1 $\beta$ secretion in RA-FLSs. Conversely, miR-498 inhibitor increased the level of TNF- $\alpha$ and IL-1 $\beta$ (Figures $3 \mathrm{~A}$ and $\mathrm{B}$ ). Additionally, RA-
FLS cell apoptosis was evaluated by western blotting and flow cytometry. Western blot assay demonstrated that miR-498 mimics decreased $\mathrm{Bcl}-2$, and increased Bax and cleaved caspase3 , whereas miR-498 inhibitor exhibited the reverse effect on Bcl-2, Bax, and cleaved caspase-3 protein levels (Figure $3 \mathrm{C}$ ). Similarly, flow cytometry showed that miR-498 mimics induced apoptosis while miR-498 inhibitor alleviated apoptosis in RA-FLSs (Figure 3 D). Thus, miR-498 suppress inflammation and promote apoptosis in RA-FLSs.

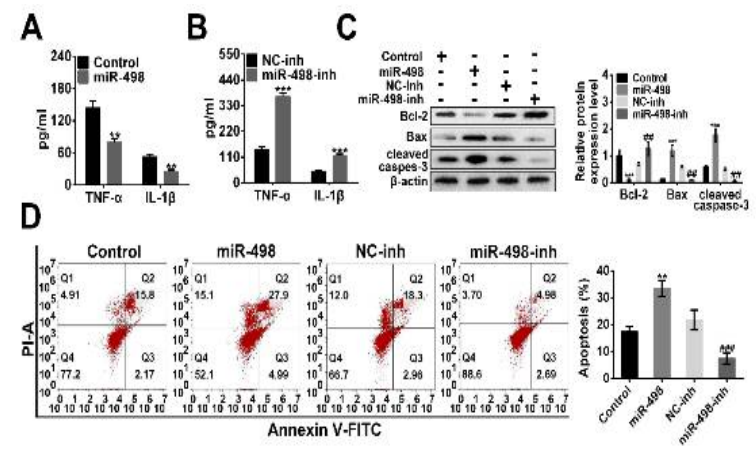

Figure 3: Effects of miR-498 on the apoptosis and inflammatory responose of RA-FLSs. (A) Inhibition of TNF- $\alpha$ and IL- $1 \beta$ in RA-FLSs by miR-498 mimics. (B) increase in TNF- $\alpha$ and IL-1 $1 \beta$ expression in RA-FLSs by miR-498 inhibitor. (C) MiR-498 mimics decreased $\mathrm{Bcl}-2$ and increased Bax and cleaved caspase-3 expressions, whereas miR-498 inhibitor promoted Bcl2 and reduced Bax and cleaved caspase-3 protein expression. (D) MiR-498 mimics induced apoptosis, whereas miR-498 inhibitor alleviated apoptosis in RAFLSs; ${ }^{* \star} p<0.001,{ }^{\# \#} p<0.01,{ }^{\# \#} p<0.001$; "comparison betweenRA-FLSs transfected with or without miR-498 mimic; "comparison between RAFLSs transfected with or without miR-498 inhibitor

\section{JAK1 is a target of miR-498}

To further explore the molecular mechanism of miR-498 in RA, it was predicted that miR-498 binds to JAK1 (Figure $4 \mathrm{~A}$ ). Dual luciferase assay confirmed that miR-498 mimics significantly reduced the relative luciferase activity in JAK1 3'UTR-wt, whereas no significant change was found in JAK1 3'UTR-mut (Figure 4 B). Moreover, western blotting revealed that miR498 mimics suppressed JAK1 protein levels while miR-498 inhibitor enhanced JAK1 levels (Figure $4 \mathrm{C}$ ). Together, these data showed that JAK1 is the target of miR-498 and its levels could be affected by miR-498.

MiR-498 regulate proliferation, inflammation, and apoptosis by inhibiting JAK1 in RA-FLSs

This study further investigated how miR-498 affected proliferation, inflammation, and 
apoptosis. MTT assay showed that miR-498 mimics inhibited cell proliferation in RA-FLSs. However, the effect was alleviated in RA-FLSs co-transfected with miR-498 mimics and JAK1 (Figure $5 \mathrm{~A}$ ). ELISA indicated that miR-498 mimics repressed TNF- $\alpha$ and IL-1 $\beta$ levels, whereas miR-498 mimics and JAK1 overexpression reversed these levels (Figure 5 $B)$. Additionally, western blotting determined that the decrease in the JAK1 and Cyclin D1 levels and the increase in p27 protein levels induced by miR-498 mimics could be reversed in the presence of JAK1. Similarly, miR-498 mimics and JAK1 overexpression reversed the reduction in Bcl-2, and the elevation of Bax and cleaved caspase-3 protein levels (Figure $5 \mathrm{C}$ ).

\section{A}
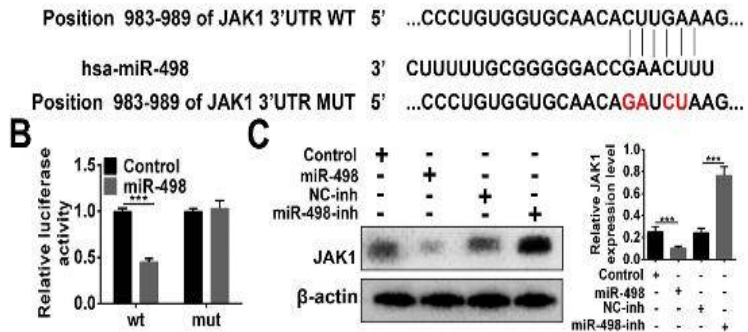

Figure 4: Effect of miR-498 on the expression of JAK1. (A) The binding site was searched in TargetScan database. (B) MiR-498 mimics significantly reduced relative luciferase activity in JAK1 3'UTR-wt, whereas no significant change was found in JAK1 3'UTR-mut. (C) MiR-498 mimics decreased JAK1 protein levels, whereas miR-498 inhibitor increased JAK1 protein levels; ${ }^{* * *} p<0.001$

Furthermore, the cell cycle was arrested in cells treated with miR-498 mimics, whereas the arrest was eliminated in cells co-transfected with miR498 mimics and JAK1 (Figure 5 D). Flow cytometry showed that miR-498 mimics augmented apoptosis, whereas miR-498 mimics and JAK1 overexpression suppressed this apoptosis (Figure $5 \mathrm{E}$ ). Taken together, miR-498 thus regulates proliferation, inflammation, and apoptosis by targeting JAK1 in RA-FLSs.

\section{DISCUSSION}

In this study, miR-498 expression was first evaluated in RA synovial tissues and RA-FLSs. To investigate the function of $\mathrm{miR}-498$ in $\mathrm{RA}$, this study carried out miR-498 overexpression or knockdown experiments in RA-FLSs. = microRNA-498 was found to inhibited proliferation, cell cycle, and inflammation, and enhanced cell apoptosis in RA-FLSs. Janus Kinase 1 was predicted to be a target of miR498. The interaction between miR-498 and JAK1 was also verified. The effect of miR-498 mimics on proliferation, cell cycle, inflammation, and apoptosis was alleviated in RA-FLSs cotransfected with miR-498 mimics and JAK1. These findings indicate that miR-498 may regulate the proliferation and inflammation of $R A$ via targeting JAK1.
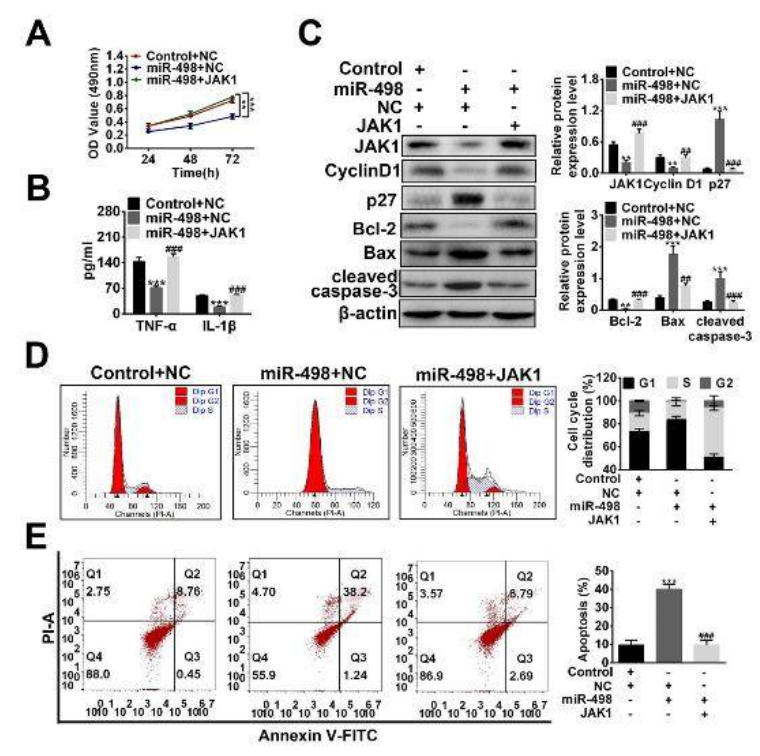

Figure 5: Effect of miR-498 on the proliferation, inflammation, and apoptosis of RA-FLSs. (A) MiR-498 mimics inhibited cell proliferation in RA-FLSs, whereas miR-498 + JAK1 cotransfection reversed this inhibition. (B) MiR-498 mimics decreased the levels of TNF- $\alpha$ and IL-1 $\beta$, whereas miR-498 + JAK1 cotransfection reversed the effect of miR-498. (C) MiR-498 mimics decrease JAK1, Cyclin D1, and Bcl-2 expressions, and increased p27, Bax, and cleaved caspase-3 expression. However, miR-498 + JAK1 cotransfection reduced these protein levels. (D) MiR498 mimics arrested the cell cycle, whereas the arrest was eliminated in RA-FLSs co-transfected with miR498 mimics and JAK1. (E) MiR-498 mimics enhanced apoptosis, whereas miR-498 + JAK1 cotransfection suppressed apoptosis in RA-FLSs; ${ }^{* *} p<0.01,{ }^{* \star *} p<$ $0.001,{ }^{\# \#} p<0.01,{ }^{\# \#} p<0.001$; ${ }^{*}$ comparison with RAFLSs transfected with or without miR-498 mimics; \#comparison with miR-498 mimic RA-FLSs cotransfected with or without JAK1

Previous evidence has demonstrated that miR498 is down-regulated in CD4+ T cells from the synovial fluid or the peripheral blood of patients with RA [17]. This study found a low miR-498 expression level in RA synovial tissues and RAFLSs. In addition, Xiang et al. showed that miR498 was associated with peripheral blood mononuclear cells from RA patients and could lead to the inhibition of cell differentiation in RA patients [18]. These data suggest that miR-498 may show a suppressive effect in RA-FLSs. Moreover, miR-498 mimics was demonstrated to inhibit proliferation, whereas miR-498 inhibitor elevated proliferation in RA-FLSs. 
Moreover, miR-498 mimics arrested the cell cycle and induced apoptosis in RA-FLSs, as evidenced by the accumulation of $\mathrm{G} 1$ cells, inhibition of Cyclin D1 and Bcl-2 expression, and enhancement of p27, Bax, and cleaved caspase3 protein expression. In addition, in allergic rhinitis patients, the level of miR-498 was enhanced and was associated with the allergic inflammation of nasal mucosa [19]. MicroRNA498 mimics could inhibit inflammation, accompanied by the reduced production of TNF$\alpha$ and IL-1 $\beta$. In contrast, miR-498 inhibitor promoted inflammation, leading to the elevation of TNF- $\alpha$ and IL- $1 \beta$ levels. Taken together, these results suggest that miR-498 may play an important role in the regulation of cell proliferation and inflammation in RA.

Accumulating evidence has shown that baricitinib, an oral selective inhibitor of JAK1, could be useful for RA treatment [20]. Furthermore, previous studies have found that suppressed of JAK1 and JAK3 phosphorylation and subsequent expressions of Stat 1 and Stat1inducible genes, thus contributing to efficient RA therapy [21]. Tofacitinib was also reported to block the IL-6 receptor, leading to JAK/STAT pathway alteration in RA [22]. On the other hand, upadacitinib, a selective inhibitor of JAK1, played an important role in the signal transduction of IL6 , as well as in interferon- $\alpha / \beta$ and interferon- $\gamma$ signal transduction [23]. Interestingly, in this study, JAK1 was inhibited in miR-498 mimic RAFLSs. JAK1 overexpression in miR-498-mimic expressing RA-FLSs reversed the decrease in proliferation as well as TNF- $\alpha$ and IL-1 $\beta$ secretion induced by miR-498 mimics. In addition, miR-498 mimics and JAK1 overexpression promoted the cell cycle and suppressed apoptosis, resulting in the reduction of cells in the G1 stage, increase in cyclin D1 and $\mathrm{Bcl}-2$ levels, and decreases in p27, Bax, and cleaved caspase-3 protein levels in RA-FLSs.

Notably, JAK1 was found to be a target of miR498 in this study. These data suggest that miR498 may function as a mediator of cell proliferation and inflammation via targeting JAK1 in RA. Taken together, miR-498 is expressed in low levels in RA tissues and RA-FLSs. miR-498 could affect cell proliferation and inflammation by targeting JAK1 in RA. This study provides new protential strategy in RA treatment. However, miR-498 may bind more target genes, which may be involved in cell proliferation and inflammation in RA. Moreover, the effect of miR-498 on the JAK-STAT signaling pathway in RA has not yet been investigated. Hence, in the future, further study will be conducted to investigate the mechanism of miR-498 regulation of cell proliferation and inflammation in RA through the JAK-STAT signaling pathway.

\section{CONCLUSION}

The findings of this work indicate that miR-498 suppressed the proliferation and inflammatory activity of RA-FLSs by targeting JAK1, thus identifying miR-498 as a promising therapeutic target for RA treatment.

\section{DECLARATIONS}

\section{Conflict of interest}

The authors declare that no conflict of interest is associated with this work. No funding was received for the work.

\section{Contribution of authors}

We declare that this work was done by the researchers listed in this article. All liabilities related with the content of this article will be borne by the authors. LC and XZ designed all the experiments and revised the paper. $\mathrm{HM}$ and FY performed the experiments. LC and XZ wrote the paper.

\section{Open Access}

This is an Open Access article that uses a funding model which does not charge readers or their institutions for access and distributed under the terms of the Creative Commons Attribution License (http://creativecommons.org/licenses/by/ 4.0) and the Budapest Open Access Initiative (http://www.budapestopenaccessinitiative.org/rea d), which permit unrestricted use, distribution, and reproduction in any medium, provided the original work is properly credited.

\section{REFERENCES}

1. Arnett FC, Edworthy SM, Bloch DA, McShane DJ, Fries JF, Cooper NS, Healey LA, Kaplan SR, Liang MH, Luthra HS et al. The American Rheumatism Association 1987 revised criteria for the classification of rheumatoid arthritis. Arthritis Rheum 1988; 31(3): 315-324.

2. Ferro F, Elefante E, Luciano $N$, Talarico $R$, Todoerti $M$. One year in review 2017: novelties in the treatment of rheumatoid arthritis. Clin Exp Rheumatol 2017; 35(5): 721-734.

3. Bax M, van Heemst J, Huizinga TW, Toes RE. Genetics of rheumatoid arthritis: what have we learned? Immunogenetics 2011; 63(8): 459-466. 
4. Guo Q, Wang $Y, X u D$, Nossent J, Pavlos $N J$, Xu J. Rheumatoid arthritis: pathological mechanisms and modern pharmacologic therapies. Bone Res 2018; 6(15).

5. Bottini N, Firestein GS. Duality of fibroblast-like synoviocytes in RA: passive responders and imprinted aggressors. Nat Rev Rheumatol 2013; 9(1): 24-33.

6. Bagnyukova TV, Pogribny IP, Chekhun VF. MicroRNAs in normal and cancer cells: a new class of gene expression regulators. Exp Oncol 2006; 28(4): 263-269.

7. Bartel DP. MicroRNAs: genomics, biogenesis, mechanism, and function. Cell 2004; 116(2): 281-297.

8. Guo HY, Cai YL, Yang SD, Jiang P. Association of MicroRNA-502-Binding Site Single Nucleotide Polymorphism of Lysine Ethyltransferase8 Gene with Cervical Cancer Risk in Chinese Women. Journal of Bionanoscience 2014; 8: 51-55.

9. Zuo J, Luo R, Huang CJ, Lou XB, Li LJ. MiR-126 enhances cisplatin chemosensitivity in hepatocellular carcinoma cells by targeting IRS1. Trop J Pharm Res 2019; 18(1): 25-30.

10. Wu J, Fan W, Ma L, Geng X. miR-708-5p promotes fibroblast-like synoviocytes' cell apoptosis and ameliorates rheumatoid arthritis by the inhibition of Wnt3a/beta-catenin pathway. Drug Des Devel Ther 2018; 12: 3439-3447.

11. Wangyang $Y$, Yi L, Wang T, Feng Y, LiU G, Li D, Zheng $X$. MiR-199a-3p inhibits proliferation and induces apoptosis in rheumatoid arthritis fibroblast-like synoviocytes via suppressing retinoblastoma 1. Biosci Rep 2018; 38(6).

12. Chatzikyriakidou A, Voulgari PV, Georgiou I, Drosos AA. miRNAs and related polymorphisms in rheumatoid arthritis susceptibility. Autoimmun Rev 2012; 11(9): 636641.

13. Westhovens R, Taylor PC, Alten R, Pavlova D, EnriquezSosa F, Mazur M, Greenwald $M$, Van der Aa $A$, Vanhoutte F, Tasset $C$ et al. Filgotinib (GLPG0634/GS6034), an oral JAK1 selective inhibitor, is effective in combination with methotrexate (MTX) in patients with active rheumatoid arthritis and insufficient response to MTX: results from a randomised, dose-finding study (DARWIN 1). Ann Rheum Dis 2017; 76(6): 998-1008.
14. Ghoreschi K, Laurence A, O'Shea JJ. Janus kinases in immune cell signaling. Immunol Rev 2009; 228(1): 273287.

15. Lou L, Zhou J, Liu Y, Wei YI, Zhao J, Deng J, Dong B, Zhu L, Wu A, Yang $Y$ et al. Chlorogenic acid induces apoptosis to inhibit inflammatory proliferation of IL-6induced fibroblast-like synoviocytes through modulating the activation of JAK/STAT and NF-kappaB signaling pathways. Exp Ther Med 2016; 11(5): 2054-2060.

16. Migita K, Komori A, Torigoshi T, Maeda Y, Izumi Y, Jiuchi $Y$, Miyashita T, Nakamura M, Motokawa S, Ishibashi $H$. CP690,550 inhibits oncostatin M-induced JAK/STAT signaling pathway in rheumatoid synoviocytes. Arthritis Res Ther 2011; 13(3): R72.

17. Li J, Wan Y, Guo Q, Zou L, Zhang J, Fang Y, Fu X, Liu H, Lu L, Wu Y. Altered microRNA expression profile with miR-146a upregulation in CD4+ $T$ cells from patients with rheumatoid arthritis. Arthritis Res Ther 2010; 12(3): $R 81$.

18. Xiang HY, Pan F, Yan JZ, Hong LQ, Zhang LH, Liu YH, Feng $X$, Cai CS. [Upregulation of miR-498 suppresses Th17 cell differentiation by targeting STAT3 in rheumatoid arthritis patients]. Sheng Li Xue Bao 2018; 70(2): 167-174.

19. Suojalehto H, Toskala E, Kilpelainen M, Majuri ML, Mitts $C$, Lindstrom I, Puustinen A, Plosila T, Sipila J, Wolff $H$ et al. MicroRNA profiles in nasal mucosa of patients with allergic and nonallergic rhinitis and asthma. Int Forum Allergy Rhinol 2013; 3(8): 612-620.

20. Choy EHS, Miceli-Richard C, Gonzalez-Gay MA, Sinigaglia L, Schlichting DE, Meszaros G, de la Torre I, Schulze-Koops $H$. The effect of JAK1/JAK2 inhibition in rheumatoid arthritis: efficacy and safety of baricitinib. Clin Exp Rheumatol 2019.

21. Tanaka Y. Recent progress and perspective in JAK inhibitors for rheumatoid arthritis: from bench to bedside. J Biochem 2015; 158(3): 173-179.

22. Alten R, Maleitzke T. Tocilizumab: a novel humanized anti-interleukin 6 (IL-6) receptor antibody for the treatment of patients with non-RA systemic, inflammatory rheumatic diseases. Ann Med 2013; 45(4): 357-363.

23. Collison J. Selective inhibition of JAK1 shows promise for RA. Nat Rev Rheumatol 2018; 14(8): 018-0054. 